\title{
Desarrollo de modelo para la capacitación de los roles funcionales
}

\author{
Alberto Escobedo Portillo \\ alberto.ep@chihuahua.tecnm.mx \\ ${ }^{1}$ Instituto Tecnológico de Chihuahua - TecNM \\ Chihuahua, Chih. México \\ Luis Arnulfo Guerrero Chávez \\ 1guerrer@itchihuahua.edu.mx \\ Instituto Tecnológico de Chihuahua - TecNM \\ Chihuahua, Chih. México \\ Luz Ernestina Fierro Murga \\ 1fierro@ulsachihuahua.edu.mx \\ Universidad La Salle Chihuahua \\ Chihuahua, Chih. México
}

\section{RESUMEN}

Los roles funcionales establecen que los seres humanos tienen características que permiten clasificarlos en tres categorías generales de roles: mentales, sociales y de acción. Por lo tanto, para que un equipo funcione de manera ordenada y sinérgica, es necesario que esté equilibrado, idealmente en partes iguales. El objetivo de este estudio es diseñar un modelo para el desarrollo de los roles funcionales. La investigación es cualitativa, con alcance descriptivo, con base en el método analítico-sintético. Se emplea la recopilación y el análisis de información escrita y tablas comparativas. Como resultado, se obtuvieron ocho factores que bien podrían ser la base del diseño de instrumentos de medición, con el propósito de diagnosticar las necesidades de capacitación y, con base en ello, generar programas de desarrollo de acuerdo con los roles funcionales. Se recomienda operacionalizar los factores identificados para aplicar y validar el modelo.

Palabras clave: roles funcionales; trabajo en equipo; modelo de desarrollo; capacitación. 


\title{
Development of a model for the training of functional roles
}

\begin{abstract}
Functional roles establish that human beings have characteristics that allow them to be classified into three general categories of roles: mental, social, and action. Therefore, for a team to function in an orderly and synergistic manner, it needs to be balanced, ideally in equal parts. The objective of this study is to design a model for the development of functional roles. The research is qualitative, descriptive, applied, based on the analyticalsynthetic method. The compilation and analysis of written information and comparative tables are used. As a result, eight factors were obtained that could well be the basis for the design of measurement instruments, in order to diagnose training needs, and based on this, generate development programs according to functional roles. It is recommended to operationalize the identified factors to apply and validate the model
\end{abstract}

Keywords: Functional roles, teamwork, development model, training

Artículo recibido: 02 noviembre. 2021 Aceptado para publicación: 28 noviembre 2021 Correspondencia: alberto.ep@ chihuahua.tecnm.mx Conflictos de Interés: Ninguna que declarar 


\section{INTRODUCCIÓN}

Un tema primordial en el quehacer diario de toda organización es la administración de sus recursos, incluida la gestión del talento humano: reclutamiento y selección de personal; establecimiento de actividades y responsabilidades; evaluación del desempeño; reuniones de seguimiento; retención, capacitación, entre tantas otras tareas. Estudios reflejan que el trabajo en equipo ha sido considerado la competencia más valorada en México (Gobierno de México, 2018; Centro de investigación para el desarrollo, 2014), lo que evidencia que los equipos de trabajo son uno de los recursos más solicitados por las empresas para afrontar los retos actuales, la incertidumbre y los entornos hipercompetitivos, es decir, saber trabajar en equipo se ha convertido en una característica personal que se considera esencial en el mundo laboral actual, debido a que los equipos "aportan diversidad de actitudes, experiencias, habilidades y conocimientos cuya integración permite ofrecer soluciones flexibles, innovadoras y rápidas a diferentes retos" (Ullah, Khattak y Rahman, 2018, p.50). La mayoría de los empleados, en algún momento de su carrera profesional tendrán que trabajar en equipo, para responder a las actividades interdisciplinarias tan demandadas por las organizaciones, lo que insta a que las empresas busquen individuos capaces de colaborar e interrelacionarse con otros, a causa de las incesantes exigencias de productividad, competitividad e innovación, entre otras, y la tendencia de hacer más, con menos, es decir, lograr sinergia (Guerrero, 2013). Sin embargo, suele confundirse la diferencia entre un grupo y un equipo de trabajo; los primeros operan sin una cultura estable y clara, de forma independiente, a diferencia de los últimos, en los cuales existe una diferenciación de las habilidades de los individuos para trabajar de manera interdependiente (Farooqi, Ashraf y Nazeer, 2020), para desempeñar tareas específicas que se añaden a los esfuerzos de los demás, para lograr un fin común (Wienclaw, 2016). Para que se dé el trabajo en equipo se necesita diferenciar las habilidades de los individuos, y "sus miembros deben estar regidos por el mismo marco normativo, que asegure su integración” (Rapimán et al. 2020, p.38) y, dado que la teoría de los roles funcionales alude a “...nuestro modo individual, personal, de comportarnos, de contribuir a la tarea y de relacionarnos con otras personas en el trabajo." (Belbin, 2010, p.15), se considera complementaria para integrar y desarrollar equipos de trabajo. 
Trabajar en equipo brinda todas las condiciones para aumentar conocimientos, aprender e innovar (Arrea, 2016), lo que enriquece la moral de los empleados. Sin embargo, no todos los equipos consiguen estos resultados (Hayes, 2003). Por su parte, Katzenbach y Smith (1996) mencionan que, para tomar buenas decisiones relacionadas con los equipos, se debe considerar que son un conjunto de personas comprometidas con un propósito, y que aportan habilidades y perfiles que se complementan.

\section{Roles funcionales}

A partir de su aparición, el tema de los roles funcionales ha sido estudiado desde distintos enfoques, bajo la influencia de diversas culturas y a través del diseño de distintos experimentos. Algunos de los primeros investigadores en iniciar la observación de grupos de trabajo fueron Benne y Sheats (1948), registrando un modelo de diecisiete roles, a partir de observar cuando individuos trabajaban unidos. Los roles funcionales, o de equipo, se refieren a “...el conjunto de características, comportamientos y habilidades que cada persona posee de manera natural y debe desarrollar, para hacerse cargo de lo que le resulta común y ventajoso". (Escobedo y Guerrero, 2013, p.17); sin embargo, no están directamente relacionados con la posición que el individuo ocupa en la jerarquía de la empresa (Arizeta, 2005). A través de este tipo de roles es posible identificar las características predominantes de los individuos, enfocándose en sus comportamientos al trabajar con otros, al igual que en sus habilidades naturales. Hay tres tipos de roles funcionales llamados genéricos (Belbin, 2017; Escobedo y Guerrero, 2016), que interactúan en los equipos de trabajo: mentales, cuya función es aportar ideas para nuevos productos y/o servicios y lo relacionado con solución de problemas y toma de decisiones; sociales, quienes coordinan esfuerzos, obtienen recursos y cohesionan al equipo; y, de acción, quienes ponen en práctica las ideas y planes propuestos por el equipo, al tiempo que se encargan del logro de las metas.

Por otra parte, existe una subcategoría de cada uno de los roles genéricos, denominada roles específicos la cual es la forma más empleada en la literatura. En la Tabla 1 se ilustra el modelo de roles de equipo desarrollado por Belbin (2010), ordenado de acuerdo con el criterio de clasificación de los roles funcionales genéricos y específicos. 
Tabla 1. Roles de equipo de Belbin

\begin{tabular}{llll}
\cline { 2 - 4 } & \multicolumn{3}{c}{ Roles genéricos } \\
\cline { 2 - 4 } & Mentales & \multicolumn{1}{c}{ Sociales } & \multicolumn{1}{c}{ Acción } \\
\hline \multirow{2}{*}{ Roles } & Cerebro & Coordinador & Implementador \\
específicos & Especialista & Cohesionador & Impulsor \\
& Monitor & Investigador de recursos & Finalizador \\
& Evaluador & & \\
\hline
\end{tabular}

Fuente: Belbin (2010)

\section{Equipos de trabajo balanceados en roles funcionales}

Un equipo balanceado es aquel que cuenta con al menos un rol mental, uno social y uno de acción, mas, balancear un equipo de trabajo supone dificultades, ya que se sabe que existe predominancia de ciertos roles en los sectores privado y público (Arroba y Wedgwood-Oppenheim, 1994), y diferencias de género (Balderson y Broderick, 1996). Asimismo, se ha suscitado discusión en torno a la correlación que existe entre el alto rendimiento y los equipos balanceados: investigadores como Partington y Harris (1999) han utilizado el modelo de roles de equipo de Belbin (2010), obteniendo una baja correlación, a diferencia de Senior (1997) y Van de Water et al. (2006), que confirmaron alta correlación entre los equipos balanceados y su desempeño, con una relación positiva cuando se integran ciertos roles.

Por otra parte, Roberts y Nason (2003) indicaron que el compromiso y las actividades para construir el conocimiento son mejoradas por medio del uso de equipos balanceados en sus roles funcionales, pero no es suficiente: otros factores tales como la falta de voluntad de los participantes afectan la actividad del equipo, lo cual podría ser una consecuencia del bajo desarrollo de las habilidades de los integrantes. Van de Water et al. (2006) diseñaron un modelo matemático para combinar o, en sus palabras: "balancear los roles en equipos", pero a causa de la complejidad de las combinaciones, la información que genera su modelo es muy difícil de utilizar de modo eficiente: "El número de posibles combinaciones en una situación práctica real, pronto puede ascender a cientos, miles, si no millones, imposible de realizar en un tiempo razonable..." (Van de Water et al., 2006, p. 14)

Por su parte, Belbin (2010) definió un concepto nacido de la variedad de los perfiles llamado "rol secundario" que se describe como la capacidad que posee un integrante del 
equipo de trabajo para desarrollar otro tipo de rol funcional, lo cual se refiere al rol que complementa el comportamiento de los individuos que, en caso de ser necesario, podría desarrollarse por medio de adiestramiento.

$\mathrm{Al}$ respecto de la capacitación de los equipos de trabajo, McEwan et al. (2017) consideran tres vías posibles: educación didáctica en aula de clase (sensibilización y teoría); talleres interactivos (discusión y reflexión) y, entrenamiento por simulación de escenarios. No obstante, sería conveniente realizar un diagnóstico con base en el planteamiento establecido por la teoría de los roles funcionales. En la industria, hay instructores que suelen tener actitudes poco optimistas respecto a los equipos, debido a la mala calidad resultante de su esfuerzo, y a pesar de su valor potencial (Guchait, Lei y Tews, 2016).

\section{ESTRATEGIAS METODOLÓGICAS O MATERIALES Y MÉTODOS}

La investigación es de naturaleza cualitativa, documental y el alcance es descriptivo, ya que aborda el diseño del modelo de desarrollo de roles funcionales basado en la literatura; el método que prevalece es el analítico-sintético, y se emplea la recopilación y el análisis de información escrita como técnica. Se consideraron diez modelos de roles funcionales, la secuencia seguida para la conformación del modelo fue la siguiente:

1. Revisión de la literatura

2. Recopilación de información sobre descripciones de roles funcionales

3. Clasificación de los roles según criterio de roles mentales, sociales y acción

4. Señalamiento de los factores más mencionados por los autores considerados

5. Clasificación y codificación de factores afines

6. Descripción de cada uno de los factores generados

\section{RESULTADOS Y DISCUSIÓN}

A partir del análisis de la información, con base en la literatura de roles funcionales, se agruparon en tablas los roles funcionales genéricos y específicos, además de temas similares (se homogenizaron las categorías), según las descripciones de cada teoría considerada. Los factores de desarrollo resultantes comunes para los tres tipos de roles mentales, sociales y de acción, se concentran en las tablas 2, 3 y 4. 
Tabla 2. Factores del modelo de roles mentales

\begin{tabular}{|c|c|}
\hline \multicolumn{2}{|l|}{ Roles mentales } \\
\hline $\begin{array}{l}\text { Objetivos } \\
\text { metas }\end{array}$ & $\begin{array}{l}\text { Sugiere nuevos objetivos y metas (Benne y Sheats, 1948). Excelentes en la } \\
\text { planificación estratégica. Ayudan al equipo a alcanzar sus objetivos } \\
\text { (Hendrickson, 2014). Da forma original a las estrategias del equipo (Mumford, } \\
\text { 2006). Bueno en la organización de nuevas actividades. Bueno para comenzar } \\
\text { cosas nuevas (MaCann y Margerison, 1995). Traen estructura y organización a } \\
\text { las ideas (Briggs, K.C. y Briggs, I., 2015). }\end{array}$ \\
\hline Innovación & $\begin{array}{l}\text { Le gusta innovar. No tiene miedo a desafiar las normas. Experimenta con nuevas } \\
\text { ideas (MaCann y Margerison, 1995). Da forma original a los procesos de trabajo } \\
\text { (Mumford, 2006). Sus ideas mejoran el rendimiento (Hendrickson, 2014). } \\
\text { Tienen ideas nuevas e innovadoras (Hendrickson, 2014); Barry, 1991; Mumford, } \\
\text { 2006; Briggs, K.C. y Briggs, I., 2015). Innovadores, inventores, altamente } \\
\text { creativos. Aportan ideas para nuevos productos. Destacan como creadores de } \\
\text { nuevos productos (Escobedo y Guerrero, 2013) }\end{array}$ \\
\hline cación & $\begin{array}{l}\text { Escuchan, hacen preguntas y absorben información (Briggs, K.C. y Briggs, I., } \\
\text { 2015). Serio (Belbin, 1993; Hendrickson, 2014). Poco ortodoxo (Belbin, 1993). } \\
\text { Les gusta trabajar solos. Son Independientes. Son introvertidos. Tienen } \\
\text { problemas para comunicarse con personas que no son como ellos (Escobedo y } \\
\text { Guerrero, 2013) }\end{array}$ \\
\hline Liderazgo & $\begin{array}{l}\text { Se enorgullecen de ser expertos y poseer amplios conocimientos técnicos } \\
\text { (Escobedo y Guerrero, 2013) }\end{array}$ \\
\hline $\begin{array}{l}\text { Clima } \\
\text { organizacional }\end{array}$ & $\begin{array}{l}\text { Comportamientos relacionados con "ir contra la corriente" (Mumford, 2006). } \\
\text { Serios y prudentes, con fuerte autocontrol (Escobedo y Guerrero, 2013) }\end{array}$ \\
\hline $\begin{array}{l}\text { Solución } \\
\text { problemas }\end{array}$ & $\begin{array}{l}\text { Promueve una mejor comprensión mediante la exploración de implicaciones } \\
\text { (Benne y Sheats, 1948). Aportan claridad. Explican el cómo y por qué (Briggs, } \\
\text { K.C. y Briggs, I., 2015). Resuelve problemas (Belbin, 1993). Son asertivos } \\
\text { dentro de su área de dominio (Mumford, 2006). Aportan ideas para solución de } \\
\text { problemas. Emiten juicios razonados considerando todos los factores. Su } \\
\text { función principal es generar propuestas y resolver problemas difíciles. Además } \\
\text { de analizar problemas, son excelentes para evaluar y sugerir nuevas ideas } \\
\text { (Escobedo y Guerrero, 2013) }\end{array}$ \\
\hline $\begin{array}{l}\text { Toma de } \\
\text { decisiones }\end{array}$ & $\begin{array}{l}\text { Astuto para tomar decisiones. Someten las ideas o las decisiones a una } \\
\text { evaluación crítica (Mumford, 2006). Consideran todos los factores } \\
\text { (Hendrickson, 2014). Evalúa las diferentes opciones (MaCann y Margerison, } \\
\text { 1995; Belbin, 1993). Se les puede tener en cuenta en la toma de decisiones por } \\
\text { su conocimiento y experiencia (Escobedo y Guerrero, 2013) }\end{array}$ \\
\hline $\begin{array}{l}\text { Revisión } \\
\text { avance }\end{array}$ & $\begin{array}{l}\text { Somete a prueba los logros (Benne y Sheats, 1948). Juzga con exactitud (Belbin, } \\
\text { 1993). Son lentos para tomar decisiones, pues reflexionan detenidamente y se } \\
\text { apoyan en su capacidad crítica. Son imprescindibles al inicio de los proyectos o } \\
\text { cuando un plan determinado no progresa (Escobedo y Guerrero, 2013) }\end{array}$ \\
\hline
\end{tabular}


Tabla 3. Factores del modelo de roles sociales

\begin{tabular}{|c|c|}
\hline Roles sociales & \\
\hline $\begin{array}{l}\text { Objetivos } \quad y \\
\text { metas }\end{array}$ & $\begin{array}{l}\text { Mantiene la orientación del grupo hacia los objetivos establecidos (Benne y Sheats, } \\
\text { 1948). Aclara las metas a alcanzar (Belbin, 1993). Orientado a objetivos y metas. } \\
\text { No dan suficiente énfasis al progreso hacia las metas (Parker, 1996) }\end{array}$ \\
\hline Innovación & $\begin{array}{l}\text { Busca nuevas oportunidades (Belbin, 1993; Hendrickson, 2014). Promueven la } \\
\text { exploración de nuevas y mejores formas de hacer las cosas para descubrir el } \\
\text { potencial oculto de las personas (Briggs, K.C. y Briggs, I., 2015). Se les facilita } \\
\text { buscar información y compartirla, con relación a ideas innovadoras o recursos que } \\
\text { se encuentran fuera del grupo (Escobedo y Guerrero, 2013) }\end{array}$ \\
\hline Comunicación & $\begin{array}{l}\text { Aclara temas claves (Benne y Sheats, 1948). Extrovertido, entusiasta. Personas } \\
\text { maduras, con seguridad en sí mismos. Desarrolla contactos. Aclara las metas a } \\
\text { alcanzar. Cooperador, apacible, perceptivo y diplomático (Belbin, 1993). Reúne } \\
\text { información y hace que sea comprensible (MaCann y Margerison, 1995). Habilidad } \\
\text { para persuadir a los demás. Saben escuchar. Son buenos comunicadores } \\
\text { (Hendrickson, 2014; Belbin, 1993). Independiente (Parker, 1996; Escobedo y } \\
\text { Guerrero, 2013). Tienen la habilidad para obtener información de otras personas } \\
\text { Escobedo y Guerrero, 2013). }\end{array}$ \\
\hline Liderazgo & $\begin{array}{l}\text { Representa al equipo (Ancona y Caldwell, 1992). Habilidad para persuadir a los } \\
\text { demás (Hendrickson, 2014). Motiva a los miembros. Entrena a los miembros del } \\
\text { equipo (Mumford, 2006). Tienen la capacidad de dirigir a grupos formados por } \\
\text { gente con diversas habilidades y características personales (Escobedo y Guerrero, } \\
\text { 2013) }\end{array}$ \\
\hline $\begin{array}{l}\text { Clima } \\
\text { organizacional }\end{array}$ & $\begin{array}{l}\text { Fomenta la solidaridad. Ayuda a impedir y resolver conflictos armonizando } \\
\text { posiciones (Benne y Sheats, 1948). Se centra en el desarrollo y el mantenimiento } \\
\text { del equipo (Barry, 1991). Le gusta ayudar a otros (MaCann y Margerison, 1995). } \\
\text { Trae ánimo al equipo (Hendrickson, 2014; Mumford, 2006). Voluntad para resolver } \\
\text { problemas personales (Hendrickson, 2014). Crean un ambiente de equipo positivo } \\
\text { (Briggs, K.C. y Briggs, I., 2015; Mumford, 2006). Ponen atención a los sentimientos } \\
\text { de los miembros, fortaleza emocional para otros (Mumford, 2006; MaCann y } \\
\text { Margerison, 1995). Inicia discusión, lucha de poder. Se ajusta a las expectativas de } \\
\text { otros (Mumford, 2006). Evitan que surjan problemas interpersonales entre } \\
\text { miembros del equipo para estimular el trabajo eficiente (Escobedo y Guerrero, } \\
\text { 2013) }\end{array}$ \\
\hline $\begin{array}{l}\text { Solución de } \\
\text { problemas }\end{array}$ & $\begin{array}{l}\text { Creación de normas referente a problemas en los procesos (Mumford, 2006). Reúne } \\
\text { ideas o sugerencias (Benne y Sheats, 1948). Procuran enfrentarse a los problemas } \\
\text { con calma (Escobedo y Guerrero, 2013) }\end{array}$ \\
\hline $\begin{array}{l}\text { Toma de } \\
\text { decisiones }\end{array}$ & $\begin{array}{l}\text { Acepta y elogia diversos puntos de vista. Alienta la participación de todos (Benne } \\
\text { y Sheats, 1948). Promueve la toma de decisiones (Belbin, 1993) }\end{array}$ \\
\hline $\begin{array}{l}\text { Revisión del } \\
\text { avance }\end{array}$ & $\begin{array}{l}\text { Registra y anota los procesos (Benne y Sheats, 1948). Coordina las acciones } \\
\text { sugiriendo seguimiento. Solicita información oportuna sobre el desempeño del } \\
\text { equipo (Mumford, 2006) }\end{array}$ \\
\hline
\end{tabular}


Tabla 4. Factores del modelo de roles de acción

\begin{tabular}{|c|c|}
\hline \multicolumn{2}{|l|}{ Roles de acción } \\
\hline $\begin{array}{l}\text { Objetivos } \quad y \\
\text { metas }\end{array}$ & $\begin{array}{l}\text { Presta especial atención a los detalles, plazos, tiempo, eficiencia, estructura y } \\
\text { cumplimiento de promesas (Barry, 1991) (Hendrickson, 2014). Le gusta trabajar } \\
\text { con información detallada (MaCann y Margerison, 1995). Le gusta terminar las } \\
\text { cosas a tiempo y dentro del presupuesto (MaCann y Margerison, 1995; Belbin, } \\
\text { 1993). Metódico (MaCann y Margerison, 1995). Sistemático con sentido de lo que } \\
\text { es viable, factible y valioso (Hendrickson, 2014). Organizan y sistematizan, } \\
\text { aplicando procedimientos (Briggs, K.C. y Briggs, I., 2015). Les preocupan los } \\
\text { intereses de la compañía por encima de los suyos, lo que propicia que sean leales a } \\
\text { ella (Escobedo y Guerrero, 2013) }\end{array}$ \\
\hline Innovación & Impulsa al equipo a seguir hacia logros mayores (Escobedo y Guerrero, 2013) \\
\hline Comunicación & $\begin{array}{l}\text { Orientado a cuestionamientos, sincero, firme, dispuesto a estar en desacuerdo } \\
\text { (Parker, 1996). Buenos oyentes. Deseo de proporcionar información libre de errores } \\
\text { (Hendrickson, 2014). Disciplinado, leal, conservador. Esmerado concienzudo y } \\
\text { ansioso (Belbin, 1993). Tiene poca paciencia con el proceso de comunicación } \\
\text { (MaCann y Margerison, 1995; Parker, 1996). Fiel, confiable, eficiente. Puede ser } \\
\text { egoísta, muy directo, inflexible (Parker, 1996). Auto motivado, enérgico, retador } \\
\text { asertivo (Hendrickson, 2014). De fuertes convicciones. Se desempeña bajo un papel } \\
\text { proactivo (Briggs, K.C. y Briggs, I., 2015). Tienden a ser "agresivos", extrovertidos } \\
\text { y con mucha iniciativa. Reaccionan a la decepción o frustración (Escobedo y } \\
\text { Guerrero, 2013) }\end{array}$ \\
\hline Liderazgo & $\begin{array}{l}\text { Estimula al grupo a seguir a logros mayores (Benne y Sheats, 1948). Retador, } \\
\text { dinámico, trabaja bien bajo presión (Belbin, 1993). Son altamente motivados, con } \\
\text { gran energía y necesidad de nuevos logros. Dirigen y empujan a otros a la acción } \\
\text { (Escobedo y Guerrero, 2013) }\end{array}$ \\
\hline $\begin{array}{l}\text { Clima } \\
\text { organizacional }\end{array}$ & $\begin{array}{l}\text { Les gusta retar a los demás y su máxima preocupación es ganar. Son obstinados y } \\
\text { enérgicos (Escobedo y Guerrero, 2013) }\end{array}$ \\
\hline $\begin{array}{l}\text { Solución de } \\
\text { problemas }\end{array}$ & $\begin{array}{l}\text { Tiene iniciativa y coraje para superar obstáculos. Busca los errores y omisiones } \\
\text { (Belbin, 1993). Encuentran la manera de superar los obstáculos. Sistemáticamente } \\
\text { enfrentan a los problemas. Apoyan el trabajo duro (Escobedo y Guerrero, 2013) }\end{array}$ \\
\hline $\begin{array}{l}\text { Toma de } \\
\text { decisiones }\end{array}$ & $\begin{array}{l}\text { Impulsa a los demás a decisiones y acciones; Dispuesto a tomar decisiones } \\
\text { impopulares (Hendrickson, 2014). Examen crítico en la decisión y el suministro de } \\
\text { insumos (Briggs, K.C. y Briggs, I., 2015) }\end{array}$ \\
\hline $\begin{array}{l}\text { Revisión del } \\
\text { avance }\end{array}$ & $\begin{array}{l}\text { Evalúa la calidad de los procesos; Aporta pruebas en los análisis y resultados (Benne } \\
\text { y Sheats, 1948). Bueno con hechos y cifras (MaCann y Margerison, 1995) }\end{array}$ \\
\hline
\end{tabular}

Los resultados del estudio indican que, a partir de ocho categorías generales, es posible integrar temas para diagnosticar capacidades, con enfoque en cada uno de los tipos de roles genéricos, mentales, sociales y de acción. A continuación, con base en el análisis anterior, se propone la definición de los factores de desarrollo generados. 
Liderazgo. Establece las características naturales del individuo que le permiten tener influencia con pares y subordinados.

Comunicación. Es el proceso mediante el cual se mantiene el flujo de la información en todos los niveles del equipo.

Objetivos y metas. Se refiere al establecimiento, orientación e implementación de la planeación. En este tema los roles funcionales sugieren, establecen, organizan, aclaran, revisan y sistematizan los objetivos y las metas del equipo.

Clima organizacional. La manera en que el comportamiento personal contribuye a crear relaciones favorables con los demás para, de esta manera, fomentar la cohesión, la cooperación y el desarrollo del equipo.

Solución de problemas. Describe los enfoques que el equipo utiliza para proponer, identificar y llevar a la práctica acciones que resuelvan situaciones de conflicto, entre personas o de situaciones técnicas y operativas.

Revisión del avance. Establece las aportaciones de las personas a la revisión periódica de los progresos, evaluando los resultados basados en métricos grupales e individuales.

Toma de decisiones. Se refiere a las interacciones realizadas por el equipo para llegar al consenso por medio de la participación de todos los integrantes.

Innovación. Propuesta de nuevas y mejores formas de realizar las cosas a través de ideas, búsqueda del desarrollo del equipo y de la exploración de alternativas.

\section{CONCLUSIÓN O CONSIDERACIONES FINALES}

Se advierte que las categorías identificadas -a partir del análisis de las teorías de roles funcionales- no sólo permiten establecer que todas las características y cualidades descritas por diez teorías afines, se complementan y enriquecen la mirada hacia los atributos y comportamiento que deben identificarse, fortalecerse $\mathrm{y}$, en su caso, desarrollarse y reconocerse entre los integrantes de un equipo de trabajo, pues el hecho de resaltar las aportaciones de cada individuo, al equipo, ayuda a magnificar el sentido profundo de la sinergia, al entender que es la unión de los eslabones, y no cada eslabón per se, lo que da y agrega valor al trabajo, pues al reconocerse como una sólida agrupación que interactúa y reconoce la función e importancia de cada persona y del colectivo encaminado a un mismo fin, la labor se torna grata y los conflictos un reto que se asume con integral compromiso. De ahí la importancia de diagnosticar si los llamados equipos de trabajo están realmente equilibrados en comportamientos, conocimientos y habilidades 
funcionales. Este planteamiento pretendió generar factores que sustenten la realización de diagnósticos de capacitación a partir del enfoque de roles, con el propósito de estimular la colaboración y la participación desde dentro de los equipos de trabajo, al tiempo que permite descubrir, bajo un enfoque racional, la forma de compartir el intelecto que conjugue emoción y saber, razón y experiencia para construir colaboración inter-equipos, estableciendo así redes de colaboración laboral que respondan y aprovechen las competencias funcionales, a partir de equipos cuyas miradas se posan en la responsable identificación de lo que cada quien aporta al todo de su trabajo y que, con mirada sistémica reconozca que cada parte contribuye a la totalidad de su labor, por ende, que las partes aisladas generan disrupción y quebranto en el objetivo pretendido.

Si bien los autores han realizado estudios de campo que les han permitido definir los roles funcionales en algunas empresas en la ciudad de Chihuahua, México, reconocen que el estudio no se agota, sino que es necesario incentivar más investigaciones, y a mayor profundidad, con el fin de comprobar que, efectivamente, las categorías definidas en este estudio orientan hacia una capacitación basada de los roles funcionales.

\section{LISTA DE REFERENCIAS}

Ancona, D., \& Caldwell, D. (1989). Improving the performance od new product teams. Sloan School o Management, 6-14.

Arizeta, A. (2005). Team Roles Preference and Conflict Management Styles. The International Journal of Conflict Management, 157-182

Arrea, C. (2016). Más sobre el trabjo en equipo. Acta médica Costarricense, 96-99.

Arroba, T., \& Wedgwood-Oppenheimer, F. (1994). Do Senior Mangers Differ in the Public and Provate Sector? An Examination of Team Role Preferences. Journal of Managerial Psychology, 9(2), 13-16.

Balderson, S. J., \& Broderick, A. J. (1996). Behaviour in teams: exploring occupational and gender differences. Journal of managerial psychology, 11, 33-42.

Barry, D. (1991). Managing the bossless team: Lesson in distributed leadership. Organizational dynamics, 21(1), 31-47.

Belbin, M. (2010). Belbin. Recuperado el Abril de 2021, de http://www.belbin.com/content/page/4432/BELBIN-MRVSR-

AComprehensiveReview-Mar2010.pdf 
Belbin, M. (2010). Management teams: why they succeed or fail. UK: Butterworth Heinemann.

Belbin, M. (2010). Team roles at work. UK: Routledge.

Belbin, M. (10 de 10 de 2017). Recursos gratuitos: Belbin. Recuperado el 11 de septiembre de 2021, de Belbin Associates: http://www.belbin.es/content/page/9264/Belbin.esEl\%20Circulo\%20de\%20Roles\%20de\%20Equipo.pdf

Benne, K. D., \& Sheats, P. (1948). Functional roles os group members. Journal of social issues, 4(2), 41-49.

Briggs, K. C., \& Briggs, I. (2015). MBTI Basics. Recuperado el Abril de 2021, de http://www.myersbriggs.org

Centro de investigación para el desarrollo. (2014). Encuesta de competencias profesionales 2014: ¿Qué buscan -y no encuentran- las empresas en los profesionistas jóvenes? México: CIDAC.

Escobedo, A., \& Guerrero, L. (2016). Desarrollo de un instrumento válido y confiable para la determinación de los roles funcionales. XXI Congreso Internacional de Administración, COntaduría e Informática. México: UNAM.

Escobedo, A., \& Guerrero, L. A. (2013). Los roles funcionales en el trabajo. Chihuahua: Hibri-Books.

Farooqi, R., Ashraf, F., \& Nazeer, I. (2020). Interpersonal Communication, Teamwork Effectiveness, and Organizational Commitment in Pakistani Nurses. Pakistan Journal of Psychological Research, 675-692.

Gobierno de México. (2018). conocer.gob.mx. Obtenido de Encuesta anual sobre competencias en México: Informe de resultados 2017: https://conocer.gob.mx/wpcontent/uploads/2018/06/informe_de_resultados_encuesta_anual_de_competenc ias_2017.pdf

Guchait, P., Lei, P., \& Tews, M. J. (2016). Making Teamwork Work: Team Knowledge for Team Effectiveness. Journal of Psychology, 300-317.

Guerrero, L. A. (2013). Modelo integral y dinámico para desarrollo de grupos de trabajo. Chihuahua: Gestoría. 
Hayes, N. (2003). Dirección de equipos de trabajo: Una estrategia para el éxito. Madrid: Thomson Paraninfo

Hendrick, T. (2014). Thomas International. Recuperado el Enero de 2021, de http://www.kiamichi-poteau.tec.ok.us/users/c/cpu/www/cpu/docs/poster.pdf

Katzenbach, J., \& Smith, D. K. (1996). La Sabiduría de los equipos. Madrid: Diaz de Santos

Margerison, C., \& McCann, D. (1995). Team management: practical new approaches. London: Management Books 2000.

McEwan, D., Ruissen, G. R., Eys, M. A., Zumbo, B. D., \& Beauchamp, M. R. (2017). The Effectiveness of Teamwork Training on Teamwork Behaviors and Team Performance: A Systematic Review and Meta-Analysis of Controlled Interventions. PLOS ONE, 1-23.

Mumford, T. (2008). The team roles test: Development and validation of a team role knowledge situational judgement test. Journal of applied psychology, 93(2), 250267.

Parker, G. (2008). Team players and teamwork: new strategies for developing successful colaboration. San Francisco: Wiley \& Sons.

Partington, D., \& Harris, H. (1999). Team role balance and team performance: an empirical study. Journal of management development, 18(8), 694-705.

Rapimán, M., Acevedo, I., Osorio, M., \& Torres, A. (2020). Paul Ricoeur y una vida buena con otros y para otros: A propósito del trabajo en equipo en salud. Acta Bioethica, 37-42.

Roberts, A., \& Nason, R. (2003). The team balancing act - enhancing knowledge building activity in on-line learning communities. Auckland: New Zealand Association for research in education.

Senior, B. (1997). Team roles and team performance: Is there "really" a link? Journal of occupational and organizational psychology, 241-258.

Ullah, R., Khattak, S. R., \& Rahman, S. (2018). The Buffering Effect of Teamwork Effectiveness on the Relationship between Employee Work Engagement and Behavioral Outcomes. Journal of Managerial Sciences, 49-61.

Van de Water, T., Van de Water, H., \& Bukmanc, C. (2006). A balanced team generating model. European Journal of Operational Research, 180(2), 885-906. 
Desarrollo de modelo para la...

Wienclaw, R. (2016). Teams \& Team Building. Research Starters Business 